\title{
PROMOTORAS DE CAMBIOS, PROTAGONISTAS DE LUCHAS: cultura politica de las mujeres en Costa Rica
}

Nancy Piedra Guillén

\section{RESUMEN}

El presente artículo trata sobre la participación político organizativa de las mujeres en Costa Rica durante el siglo XX. Parte de las teorías de la cultura política con el objetivo de hacer un rescate histórico sobre la presencia e influencia de las mujeres costarricenses en el acontecer socio político del país, particularizando en los espacios propios que promueven las mujeres a lo largo del siglo, con el fin de promover y defender los derechos de las mujeres.

\section{ABSTRACT}

This article is about the political and organizational participation of women in Costa Rica during the $20^{\text {th }}$ century. It is based on the theories of political culture and aims to rescue the presence and influence Costa Rican women have had on the social and political level. It analyses, particularly, the social spaces women have been encouraging throughout the Century in order to promote and defend their rights.

\section{INTRODUCCIÓN}

En el siguiente trabajo desarrollaremos una reflexión alrededor del tema de la cultura política de las mujeres costarricenses. Dicho trabajo intenta hacer una reflexión en torno a la conceptualización del término, para posteriormente escoger una de las perspectivas analíticas que nos permita analizar el caso costarricense. Así mismo señalaremos cuáles son las principales críticas que las feministas académicas hacen al concepto, y la utilización analítica que se ha hecho desde las ciencias sociales en relación con la participación política de las mujeres. Finalmente, caracterizaremos de forma general cuál ha sido esa participación política en Costa Rica, en el siglo XX.

\section{Conceptualización de cultura política}

De forma muy general podemos plantear que cultura política es el análisis del comportamiento político de las personas (Cisneros et. al.; 1994). Sin embargo, las perspectivas analíticas son 
diversas, se diferencian entre sí, dado el acento que unos ponen en lo cultural, en lo político o en lo social. En este apartado se desarrollan algunas diferencias conceptuales relevantes, para luego desde una perspectiva analítica adecuada rescatar la participación política de las mujeres de tal forma, que las eleve como sujetas sociales. Así se contribuye a hacer visible su presencia histórica, negada por un acento androcéntrico en el conocimiento científico social.

G. A.. Almond y S. Verba son pioneros en la conceptualización de la cultura política. En esta tradición hay una especie de esmero por conceptualizar la condición humana, "desde una perspectiva racionalista de la acción" (ídem; 11).

Más específicamente estos autores pensaban la "cultura política en términos de patrones de orientación que abarcan aspectos emocionales y actitudinales respecto al funcionamiento del sistema político" (ibídem; 21). Esta conceptualización que relaciona cultura con la actividad racional de las personas ha sido duramente criticada y ha dado origen a una amplia polémica.

Por otra parte tenemos que el concepto de cultura en Alemania tiene una trayectoria histórica que se remonta a los aportes de Johann G. Herder (1744-1803). Retomando aspectos que fueron planteados en siglos pasados, los teóricos contemporáneos rescatan conceptualizaciones desarrolladas en siglos pasados y han integrado aspectos al debate como el de cultura y civilización, subjetividad social y cultura política, política comparada y cultura cívica; aspectos que a su vez complejizan el debate sobre cultura política. La complejidad del término se deriva de la amplitud y de la diversidad conceptual que existe en cada uno, el de cultura y el de política.

Samuel Beer en 1958 introdujo variables al término formulado por Almond como: valores, creencias y actitudes. Variables que debían ser analizadas dentro de una perspectiva cultural de la política. En 1961 Macridis se plantea estudiar la cultura política: metas y reglas aceptadas. Mientras Finer (1961) considera que la cultura política de un país está dada a partir de la 
legitimación de las reglas, las instituciones políticas existentes y el procedimiento. Mientras para Pye los indicadores objeto de estudio son: área de la política, los fines y significados (ídem).

Así mismo en 1965 Pye y Verba plantean que las actitudes, los sentimientos y cogniciones, respecto al sistema político proporcionan un orden y significado a los procesos políticos que conforman a su vez la cultura política (ídem). Esta perspectiva avanza hacia una propuesta analítica que relaciona lo micro con lo macro, entendido dicho puente entre la conducta política de las personas con el fenómeno político de una determinada sociedad. Como podemos ver, el concepto de cultura política que se ha abordado hasta el momento gira en torno a la actitud de las personas frente al sistema, o cómo el sistema político define la conducta política de éstas.

Robert Dhal en 1966 plantea cuatro aspectos más que deben ser integrados al análisis para abordar las orientaciones políticas de las personas con respecto al sistema, estas son: la toma de decisiones, la acción colectiva, el sistema político y la relación de la gente respecto de otros.

Por otra parte Almond y Powel introducen seis aspectos más que le dan al concepto una mayor amplitud pero que no cambia la definición del concepto, estos son: la expresión de modelos y patrones particulares que pueden existir en una cultura como los grupos étnicos, regionales o clases sociales. La cultura política como regulador del sistema. Las diferenciaciones estructurales y culturales producto del proceso de modernización y desarrollo. Enfatizar en las actitudes individuales. La socialización política que se da y que permite la reproducción de roles (ibídem: 22). Y en 1980 Almond y Verba hacen una revisión retrospectiva del concepto depurando aspectos metodológicos, pero sin "criticar el modelo racionalista y liberal de acción que sustenta el corpus explicativo de la cultura política" (ibídem: 23).

Por otra parte Rosenbaum en 1975 plantea criterios diferenciadores con respecto a los conceptos 
de estabilidad e inestabilidad, planteó así una clasificación de cultura política que va de la fragmentada a la integrada pasando por fases intermedias como: conflicto, violencia e inestabilidad (ídem).

De las corrientes explicativas planteadas hasta el momento, podemos compartir algunas inquietudes con Cisneros, Sánchez y Badie en tanto no debemos identificar, ni reducir la experiencia diferenciadora a la que hace alusión la cultura política, a lo que expresan los reportes de encuestas de opinión (ibidem:24). Así mismo los autores critican los esquemas inmediatistas centrados en la legitimación de las reglas que regulan el sistema político, desde un horizonte comparativo a partir del ideal de estabilidad y que el modelo de sujeto no agota la riqueza del análisis sobre la subjetividad social.

Estas críticas las podemos compartir siempre y cuando no dejemos de ver el avance en términos del conocimiento adquirido a partir de la preocupación tan válida que tuvieron los estudiosos de la cultura política, y que indudablemente vino a enriquecer el análisis que existía hasta el momento sobre los hechos políticos.

Hecho este rápido repaso podemos acercarnos a las propuestas de cultura política que nos acercan a nuestro tema central: la cultura política de las mujeres. Compartimos así con Cisneros, Sánchez y Badie la necesidad de integrar al análisis de la cultura política:

"las ideas de pluralidad, especificidad y diversidad de las culturas, sin perder de vista la unidad de lo diverso" (ibídem: 31).

Debemos de partir de que todo acto de razón involucra un acto de sentir, pero que históricamente se ha visto como conductas humanas contrapuestas. De igual forma que se tiende a contraponer 
razón y sentimiento, se contrapone el espacio privado al público. El espacio privado hace referencia a lo personal, a lo íntimo, a la esfera de la familia y la persona. Y lo público se relaciona con el ejercicio de la política y el trabajo. A su vez, estos ámbitos se relacionan con la división sexual del trabajo en la cual a las mujeres se las identifica con el mundo privado y a los varones con el mundo público. Una dedicada por lo tanto a la reproducción y el otro a lo productivo. Estas divisiones han hecho que a las mujeres se las relacione con actitudes pasivas y conservadoras desde los análisis de la cultura política, porque no se ve en el ámbito público con la misma fuerza y presencia de los varones. Y a los varones como los promotores del cambio y dueños del mundo público.

Estos elementos los desarrollaremos más en el apartado siguiente, en este lo que nos interesa señalar es como se ha dicotomizado el análisis de la cultura política, y cómo una propuesta analítica que tienda a relacionar el espacio público y el privado, como lo plantean Agnes Heller y F. Feber nos permitirían acercarnos a un análisis más complejo y amplio de las actitudes políticas de las personas,

"la afectividad en la vida cotidiana en tanto ámbito privado, no está alejada de lo político. Esto es, vida privada y espacio político no se encuentran desligados, dado que se traducen en consenso o disenso frente a la construcción de formas valorativas y legitimadoras de la cultura política" (ibídem: 28).

En este caso debemos también tener en cuenta los peligros que nos plantearían un mal manejo de la relación entre lo público y lo privado, tal como lo plantea Fernando Mires al señalar que no todo lo privado es político, pero todo puede ser politizado, la politización dependerá de las circunstancias que no están pre-determinadas. Esta afirmación nos lleva a plantearnos como lo político no es cerrado o es algo que está dado, son las personas las que pueden determinar 
también que es político y que no. Algo se transforma en político cuando es llevado a la política, y lo político admite la transacción y el compromiso (Mires; 1994: 93).

Sobre el uso de la expresión de que todo es político, veamos el planteamiento que el autor hace:

"Lo privado es político fue uno de los gritos de batalla de los movimientos feministas de los 70. Quería por cierto, afirmar, que la dominación patriarcal debía ser combatida en los propios hogares. Pero si lo privado es político, quiere decir, en tanto lo político es público, que lo privado es público, lo que es un contrasentido muy grande. Más como también es cierto que la dominación privada del patriarca debe ser también un tema político, lo que debe decirse en ese sentido no es que lo privado es político, sino que hay en los más diversos sentidos de la vida, temas que pueden y deben ser politizados" (Mires; 1994: 93).

Teniendo en cuenta esta aclaración sobre como debe de diferenciarse lo público, de lo privado, de lo político, sí deseamos resaltar que tomar aspectos que suceden en el ámbito privado enriquecen definitivamente el análisis de la cultura política en tanto nos acerca a un análisis más inclusivo y pluralista y por tanto más democrático.

\section{CULTURA POLÍTICA DE LAS MUJERES}

\section{Critica a los análisis tradicionales}

Estudios que se han realizado recientemente plantean como los análisis que se hacen sobre la cultura política de las mujeres tienen un fuerte sesgo que impide se analice objetivamente la participación política de las mujeres Moran (1990); Diez (1990), Rodríguez, et. al. (1990), Astelarra (1991), De Oliveira (1991), Ardaya (1994).

Los estudios de la cultura política parten de que el fenómeno no es uniforme, aunque existen características que se comparten, hay características específicas que corresponden dados los 
sectores sociales a los que pertenecen. El sexo, es considerado una variable más dentro de estos análisis con el fin de determinar diferencias.

"Ya sea que se analice el comportamiento electoral, la ubicación ideológica, la preferencia por determinados partidos, o la actitud hacia la política, o la participación en distintos espacios que competen a la política, se afirma que existen diferencias significativas entre las mujeres y los varones" (Astelarra; 1991: 71).

Desde nuestro punto de vista, no hay problema en que se diferencie la participación política de las mujeres con respecto a la de los varones. En este caso el problema se centra por la forma en que se analiza el problema, la cual desde las ciencias sociales presenta un fuerte sesgo androcéntrico Randall (1982), Sojo (1985). Dicha perspectiva analítica considera, entre otras cosas, la conducta masculina como parámetro de la "normalidad" política. Se parte del supuesto que lo masculino es lo normal, y los comportamientos de las mujeres que no fueron iguales a la de los hombres son considerados desviaciones. Este problema abarca no solo la interpretación que se hace de los datos, sino que tiene su origen en la forma en que se recolecta la información,

"En general, la variable sexo se incluye en cuestionarios diseñados para interpretar la conducta masculina, sin sostener, ni siquiera como hipótesis, que el universo político femenino puede ser diferente. Lo curioso, es que no se niegan las diferencias, sino que no se las interpreta convirtiendo al sexo en una expresión social. Al hacerlo así, la variable sexo deja de ser sociológica y, se quiera o no, se recurre sólo a su base biológica" (Astelarra; 1991: 72).

En este caso nos enmarcamos desde la perspectiva de análisis que intenta estudiar la participación política de las mujeres a partir de que estas no comparten necesariamente la misma realidad política de las mujeres. Es más, como veremos más adelante, la participación política incluye su experiencia práctica cotidiana, su realidad y es parte también del proceso de socialización, por ello suelen participar en espacios en que se abordan aspectos relacionados con las necesidades materiales, físicas y psicológicas de las personas.

Así mismo, Ardaya plantea que la participación de las mujeres en puestos públicos debe ser analizada con una visión crítica, ya que las mujeres se han incorporado en distintos espacios 
políticos, en América Latina a partir del proceso democrático, pero ello no implica que se estén desarrollando políticas y programas que tengan una visión integral, y progresista en relación con las mujeres. También se han abierto canales estatales para el tratamiento de la temática de género a través de ministerios, secretarías, subsecretarías y direcciones de la mujer, sin embargo, estos responden más a la lógica estatal que a las necesidades de la intervención política específica y diferenciada (Ardaya; 1994:73). Estos espacios pueden ser reproductores del mismo sistema, más que integradores de una perspectiva más crítica e inclusiva hacia las mismas mujeres.

Por ello es fundamental entender que los componentes estructurales y simbólicos de la realidad de las mujeres es distinta de la de los varones. Si no se toman en cuenta estas diferencias, es muy fácil reproducir los sesgos que se derivan de los análisis tradicionales, tales como: la inferioridad social de las mujeres que está implícito en los análisis androcéntricos; el acento en el fetichismo de la familia; y la tendencia a juzgarla por parámetros masculinos (Idem).

En relación con la inferioridad social de las mujeres se suele plantear que estas no se aproximan al ideal de ciudadano democrático. Ya que su nivel de conocimiento político es inferior al de los varones, esta carencia en su formación política, desde los análisis tradicionales, se explica por la falta de un nivel educativo adecuado y la falta de una socialización política, como sí la tienen los varones (Astelarra; 1991:72).

En un mundo en que todo está determinado por la conducta masculina, la participación de las mujeres en espacios públicos se convierte en un reto, ya que aunque no se tenga conciencia de género o se sea feminista, cuando se participa en ellos (en los espacios públicos) se producen cambios, por eso la resistencia de los varones es tan fuerte, y por ello se desarrollan tantos 
mecanismos reguladores, y, aunque la mayoría de las mujeres que cumplen cargos públicos no tienen una actitud crítica, la presencia de estas es amenazante para el sistema.

\section{BREVE ESBOZO DE LA PARTICIPACIÓN POLÍTICA DE LAS MUJERES EN COSTA RICA}

Los estudios que abordan el tema de la cultura política de las mujeres suelen trabajar alrededor de tres ejes: La participación política de las mujeres desde el movimiento de mujeres, el acceso a puestos de elección popular o poder y la actitud política de las mujeres con respecto a la política. Dado que en nuestro país no se han hecho trabajos que planteen el tercer aspecto, nos centraremos en el análisis de la cultura política en cuanto a la actitud de esta hacia la participación. En este caso nos interesa analizar la participación de las mujeres con respecto a algunos eventos políticos.

\subsection{Por el derecho a la ciudadania: las sufragistas se organizan}

Uno de los acontecimientos de mayor transcendencia en la vida política de las mujeres costarricenses fue la lucha por el derecho al sufragio, es decir por el reconocimiento de uno de los derechos básicos de la ciudadanía política de las mujeres ${ }^{1}$. A las mujeres se les privó de este derecho, al no ser consideradas como ciudadanas dentro de la propuesta constitucional liberal de nuestro país.

\footnotetext{
1 El concepto de ciudadanía ha estado presente en la historia de la humanidad, por dicha razón el contenido de este concepto ha variado a lo largo de los siglos, (desde la época de los griegos) "y ha cobrado nuevos contenidos en lo que se ha dado en llamar la ciudadanía moderna, que surge en los siglos XVIII y XIX según los países, y que marcan el paso de una sociedad estamental a una sociedad moderna". (Vargas, 1998: 10 doc. inédito). La ciudadanía dio estatus a cierto sector de la sociedad, pero a su vez excluyó a grandes mayorías que no cuentan con las características que definen a un ciudadano, de acuerdo a los cánones de la época moderna. Ese fue el caso específico de las mujeres, quienes a pesar de la participación activa en la lucha contra el esclavismo, o bien a favor de los derechos ciudadanos, quedaron excluidas de dichos procesos de liberación, autoafirmación de los pueblos y surgimiento de los estados nación.
} 
El movimiento sufragista se desarrolló en Costa Rica durante el siglo XIX y XX. Fue una propuesta ideológicamente liberal y la primera expresión organizativa feminista en Costa Rica. El principal objetivo de las feministas liberales era reducir o eliminar las diferencias entre hombres y mujeres:

"Subyace a esta concepción la visión de los seres humanos como individuos que comparten una esencia común: La capacidad propia de su condición humana, sustentada en la teoría política liberal" (Lara, 1994:30).

La corriente liberal plantea la búsqueda de la igualdad social de las mujeres en el interior del sistema político existente, mediante el desarrollo de proyectos de transformación de la legislación. Este principio motivó a un grupo de mujeres a luchar por el derecho del voto en Costa Rica.

La lucha por el voto femenino en Costa Rica se remonta a acontecimientos que se sucedieron desde finales del siglo XIX. Sin embargo, los hechos que se dieron en la primera mitad del siglo $\mathrm{XX}$ fueron los antecedentes inmediatos al otorgamiento del derecho del voto a las mujeres. El movimiento de la época utilizó varias modalidades políticas para lograr su objetivo, por ejemplo: las solicitudes formales ante la Asamblea Legislativa, organización de marchas y de manifestaciones de protesta, y el desarrollo de un fuerte debate en los medios de información más importantes de la época (en especial los periódicos).

En 1923 se fundó La Liga Feminista Costarricense (LFC). Esta organización fungió como la sede nacional de la Liga Internacional de Mujeres Ibéricas e Hispanoamericanas. La mayoría de las integrantes de la Liga fueron mujeres profesionales dedicadas principalmente al Magisterio. En el cargo de presidenta se nombró a Angela Acuña ${ }^{2}$ (Barahona; 1994:63).

En 1925 La LFC solicita el otorgamiento del derecho al sufragio de las mujeres ante el Congreso de la República. Esta solicitud fue apoyada por el discurso del presidente de la República, Ricardo Jiménez; no obstante su solicitud no fue aprobada. A esta solicitud le secundarán otras que de igual forma fueron rechazadas, a pesar de presentar la propuesta del derecho al voto diferenciado (en 1931 al Congreso, en 1936 al Congreso Constitucional, en 1939 a la Comisión de Legislación

2 En la Liga Feminista y en otros espacios de protesta y participación política también sobresalen: Carmen Lyra, Ana Rosa Chacón, Lilia González, Vitalia Madrigal y Victoria Madrigal, entre otras (Barahona, 1994:63). 
del Congreso $)^{3}$.

Finalmente en 1949 se logra que se le otorgue a las mujeres el derecho de voto y el derecho a ser electas. Es importante destacar el hecho que dicha lucha se desarrolla a nivel mundial, creándose un movimiento internacional del cual Costa Rica no quedó excluido. En 1918 las mujeres noruegas ganan el derecho al voto, fue el primer país en introducir el sufragio formal en la constitución política de dicho país. Después de la Primer Guerra Mundial y de la Revolución Rusa otros países introducen ese derecho de las mujeres, tal es el caso de Alemania, y en 1918 en Ecuador se otorga el derecho al voto de las mujeres, siendo el primer país de América Latina en reconocerlo (Lycklama, Geertje; Vargas, Virginia y Wieringa, Saskia: 1998).

En el reconocimiento del derecho al voto de las mujeres fueron determinantes las luchas que protagonizaron las mujeres de la época, en especial, las luchas desarrolladas por la Liga Feminista. Las mujeres que participaron en la Liga Feminista además de ser pioneras en la lucha por la defensa de los derechos de las mujeres, fueron mujeres que abrieron el camino en la consecución de derechos para las mujeres, acumularon experiencias y conocimientos que se difundieron en toda la sociedad y lograron tener alguna influencia en los partidos políticos de la época. No es casual que años después en el seno del Partido Liberación Nacional se constituyera una Secretaría de la Mujer, la cual iría paulatinamente ganando espacio y poder al interior del mismo.

La Liga no logró conformarse en un movimiento social de mujeres debido a que su reivindicación principal giró en torno al derecho del voto, y una vez obtenido este, su dinámica organizativa descendió notablemente hasta llegar a desaparecer. Algunas de sus integrantes se incorporaron a otros espacios como los partidos políticos o espacios relacionados con sus actividades laborales. Así mismo no logró acercar a otros sectores sociales de mujeres, sus integrantes eran de sectores medios, altos y profesionales.

\footnotetext{
3 El voto diferenciado hace alusión a aquellas personas -mujeres y varones- que cuentan con un desarrollo profesional y laboral como: "profesionales universitarias, profesoras del Estado, bachilleres y normalistas, las peritas mercantiles y agrícolas, las maestras de certificado superior elemental, y de idoneidad, las profesoras de arte doméstica tituladas, las profesoras de arte, las contabilistas, taquígrafas y mecanógrafas tituladas, las asistentes sanitarias, las enfermeras y obstétricas tituladas, las telegrafistas tituladas, las mujeres graduadas en colegios particulares, las mujeres que hablen dos o más idiomas del propio, las dueñas y directoras de instituciones industriales, comerciales y agrícolas" (Barahona, 1994: 106).
} 
Sin embargo, a pesar de las limitaciones que señalamos debemos destacar la importancia política de dicho movimiento y la capacidad de acción e influencia política que tuvo. La lucha por el derecho al voto es una de las luchas más importantes que las mujeres han protagonizado para ser reconocidas como ciudadanas del mundo, generando para ello un movimiento mundial que se hizo sentir en la mayoría de los países occidentales, y que introdujo cambios en la vida política de los mismos.

A pesar de que el derecho al voto y a ser elegidas fue otorgado hace 40 años, aún la desigualdad en la escena política es visible, ello lo podemos observar en los bajos porcentajes de participación que han tenido las mujeres en instancias de poder estatal. Lo que indica que la participación mayoritaria de las mujeres se encuentra en las bases, ello a pesar de sus altos niveles de preparación, amplio conocimiento, experiencia política y participación activa en la vida política de los partidos mayoritarios y minoritarios del país.

\begin{tabular}{|l|l|l|l|}
\hline PERIODO & $\begin{array}{l}\text { MINISTERIOS } \\
\text { TOTAL }\end{array}$ & MUJERES & PORCENTAJE \\
\hline $1958-1962$ & 12 & 1 & 8.3 \\
\hline $1962-1966$ & 12 & 0 & 0.0 \\
\hline $1966-1970$ & 12 & 0 & 0.0 \\
\hline $1970-1974$ & 12 & 0 & 0.0 \\
\hline $1974-1978$ & 13 & 1 & 7.7 \\
\hline $1978-1982$ & 13 & 4 & 30.8 \\
\hline $1982-1986$ & 13 & 0 & 0.0 \\
\hline $1986-1990$ & 19 & 1 & 5.3 \\
\hline $1990-1994$ & 20 & 2 & 10.0 \\
\hline $1994-1998$ & 20 & 2 & 10.0 \\
\hline & & & \\
\hline
\end{tabular}

Fuente: Camacho, Rosalía et al. Las cuotas mínimas de participación de las mujeres: Un mecanismo de acción afirmativa. Centro de Desarrollo Nacional para la Mujer y la Familia, 1996: 32.

\begin{tabular}{|c|c|c|c|}
\hline PERIODO & $\begin{array}{lr}\text { TOTAL } & \text { DE } \\
\text { DIPUTADOS } & Y \\
\text { DIPUTADAS } & \\
\end{array}$ & TOTAL MUJERES & PORCENTAJE \\
\hline 1958-1962 & 45 & 3 & 6.7 \\
\hline $1962-1966$ & 45 & 2 & 4.4 \\
\hline $1966-1970$ & 57 & 1 & 1.8 \\
\hline $1970-1974$ & 57 & 3 & 5.3 \\
\hline 1974-1978 & 57 & 4 & 7.0 \\
\hline 1978-1982 & 57 & 4 & 7.0 \\
\hline 1982-1986 & 57 & 5 & 8.8 \\
\hline $1986-1990$ & 57 & 7 & 12.3 \\
\hline
\end{tabular}

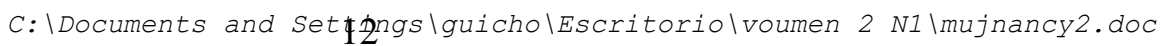




\begin{tabular}{|l|l|l|l|}
\hline $1990-1994$ & 57 & 7 & 12.3 \\
\hline $1994-1998$ & 57 & 9 & 15.8 \\
\hline & & & \\
\hline
\end{tabular}

Fuente: Camacho, Rosalía et. al. Las cuotas mínimas de participación de las mujeres: Un mecanismo de acción afirmativa. Centro Nacional para el Desarrollo de la Mujer y la Familia, 1996, p.34.

Estos datos muestran las dificultades que tenemos las mujeres para ser incluidas en el espacio público como ciudadanas, por tal razón es que en la presente década el tema y la problemática del derecho a la ciudadanía ha sido retomada por el movimiento de mujeres nacional e internacional, con el fin de introducir prácticas que posibiliten el ejercicio equitativo de la ciudadanía. Motivo por el cual, en la presente década, se revive con especial interés los logros del movimiento sufragista de principios de siglo, y se retoman algunas de las preocupaciones que en su momento fueron planteadas por dicho movimiento, como es el debate del universalismo versus la diferencia.

\subsection{Alianza de Mujeres Costarricenses: Construyendo alternativas organizativas para las mujeres del pueblo}

En 1948 se fundó la Unión de Mujeres Carmen Lyra. Esta organización estaba afiliada al Partido Vanguardia Popular. Por este motivo, fue creada en medio de la ilegalidad en que funcionó el PVP en esos años. En 1952 la Unión pasó a denominarse Alianza de Mujeres Costarricenses (AMC). Según el PVP el cambio del nombre era necesario debido a que el nombre anterior no reflejaba los verdaderos fines de la agrupación, a saber: hacer de la organización un movimiento de mujeres amplio:

"A pesar de que uno de sus objetivos principales era la defensa de los derechos de la mujer, durante catorce años la AMC funcionó dependiendo del Partido, realizando actividades en torno a la búsqueda de solidaridad internacional, apoyo a campañas mundiales, a huelgas y luchas obreras nacionales..."4 (García y Gomáriz 1989:204).

Al estar la organización afiliada al Partido Comunista se planteó como primer eje articulador de trabajo la organización de las mujeres a partir de su condición de clase y la búsqueda de la trans-

\footnotetext{
¡Error! No se encuentra el origen de la referencia. ¡Error! No se encuentra el origen de la referencia. ${ }^{4}$ García, Ana Isabel y Gomáriz, Enrique. Mujeres Centroamericanas. Tomo II, San José-Costa Rica, FLACSO, CSUCA, Universidad para la Paz, 1989. pág. 204.
} 
formación social. Y como segundo eje la lucha de las mujeres en tanto sector social discriminado.

Desde la perspectiva marxista, compartida por la Alianza de Mujeres en esa época, la opresión que viven las mujeres en la sociedad capitalista está dada por la opresión de las mujeres como fuerza de trabajo asalariada. Al igual que en el caso de los trabajadores asalariados, la alienación de las mujeres se definía en función de su inserción subordinada en el proceso productivo. De ahí que lo verdaderamente estratégico era promover la organización de la clase trabajadora y apoyar sus luchas.

Durante la década del sesenta y setenta, la Alianza de Mujeres se convirtió en la principal organización de mujeres existente en el país. Durante los setenta logró organizar y movilizar a las mujeres de la zona bananera. Sus ejes de lucha giraban en torno a la vivienda, el alza en los salarios, el control de precios en los productos de primera necesidad y la instalación de guarderías infantiles para que las mujeres pudieran incorporarse a las actividades productivas y así mejorar sus condiciones de vida (Gomáriz y García, 1989:210). A pesar de que se plantea la realización de un trabajo con mujeres, sus eje reivindicativo no partía de una perspectiva de género, sino de la importancia estratégica que tenía el sector, por ser mayoritario y por mostrar una gran capacidad de lucha y organización.

De lo planteado se deduce que como expresiones organizativas concretas de las mujeres durante el período 1940 al 70 sobresale en primer lugar la experiencia organizativa y política de la Liga Feminista Costarricense, expresión que impactó e influyó la vida política del país, y en segundo lugar la constitución de la Alianza de Mujeres Costarricenses, cómo una expresión organizativa de mujeres comunistas y mujeres de sectores populares.

Ambas experiencias político organizativas, aunque diferentes, constituyen el referente histórico en el proceso de configuración del movimiento de mujeres costarricenses. Sin embargo, no se puede perder de vista que dichas agrupaciones no fueron las únicas experiencias en las que las mujeres tuvieron una participación organizativa destacada.

Debemos partir del hecho histórico de que las mujeres en nuestra sociedad siempre han estado organizadas en múltiples espacios y en infinidad de experiencias, su presencia ha sido importante aunque los relatos y estudios históricos no den fe de dicha participación en el espacio privado ni 
en el público. También cientos de mujeres participaron en otros espacios como sindicatos, cooperativas, asociaciones comunales y partidos políticos. Dichos espacios se caracterizaron, en general, por ser espacios mixtos en los que las mujeres tenían una participación importante, aunque con pocas posibilidades de asumir cargos de dirección, y en las cuales las actividades organizativas y reivindicativas no consideraban las demandas específicas de las mujeres en tanto tales.

\subsection{Mujeres del Partido Liberación Nacional: Construyen su espacio interno}

A principios de los 60 s se inicia el movimiento de mujeres al interior del Partido Liberación Nacional (PLN). Dicha organización sólo existió por un año. Su fundación tuvo un claro carácter coyuntural: captar votos de las mujeres para las elecciones de 1962. Desde 1951 hasta fines de los sesenta la organización de las mujeres del PLN estuvo bajo la dirección de la esposa del candidato de turno; su finalidad era la de potenciar el voto femenino. A finales de la década del sesenta Karen Olsen (siendo la Primera Dama de la República) funda la Acción Femenina de Evolución Social (AFES): su fin era organizar a las mujeres del PLN para potenciar el desarrollo de las comunidades.

Las propuestas políticas de las mujeres del PLN fueron tomando cuerpo poco a poco. Su objetivo no era solo apoyar al Partido en la consecución de votos o en la realización de actividades de bienestar social, sino que se empezó a abordar el tema de la participación política dentro del Partido y el derecho a tener representación en cargos públicos.

En 1979 se organiza el Movimiento Femenino del Partido Liberación Nacional. Este movimiento promueve la inserción de las mujeres en el aparato electoral del Partido y lograr escalar puestos en el Congreso y otras Instituciones del Gobierno. Este es un hecho de gran transcendencia ya que contribuyó a gestar un mayor acercamiento entre las militantes-feministas de la época. Dicho proceso de acercamiento posibilitó, a su vez, la construcción de propuestas políticas que llegaron a transcender los intereses políticos partidarios.

Sin embargo por sí mismas no lograron durante los 60 y 70 constituirse en movimiento social de mujeres, debido a que sus actividades sólo acercaban a las militantes del Partido, circunscribiéndose su acción al ámbito de la lucha por los derechos políticos y muy particularmente por el acceso a puestos de poder al interior del PLN. 
Su estrategia organizativa no consideró como fundamental la organización de las mujeres como sujetas sociales. No consideró el desarrollo de acciones articuladas con otras agrupaciones de mujeres en función de la defensa de los derechos de las mujeres y lucha por el mejoramiento de sus condiciones de vida y en contra de los factores estructurales que generan las desigualdades de género y la discriminación contra la mujer. Sin embargo, en la década del 80 logra articularse con el movimiento de mujeres que empezó a gestarse y desarrollarse, contribuyendo a su vez a la constitución del mismo.

Durante la década del 70 promovió actividades importantes en pro de los derechos de las mujeres, son gestoras del Centro Nacional de Desarrollo de Mujer y Familia (CMF) (hoy día Instituto Nacional de la Mujer-INAMU), creado en 1976 y el "Proyecto de Ley de Igualdad Real" (1988). En el proceso de creación del CMF hay una influencia importante del contexto internacional. Es el período en el que se declara el Año Internacional de la Mujer por las Naciones Unidas y la Década de la Mujer (1975-1985), así como la Conferencia Mundial de la Mujer realizada en Nairobi (1985). Al respecto se expresa de la siguiente manera Irma González funcionaria del CMF desde sus inicios:

"La ley de creación del CMF nace en el contexto del año Internacional de la Mujer, 1975, ese año se declara el año Internacional de la Mujer que hace la primera conferencia mundial de la mujer en México y ahí salen una serie de propuestas para mejorar la condición de la mujer. Por supuesto la perspectiva era otra, los fundamentos son otros en virtud de lo que se persigue es una igualdad sin entrar a profundizar cuál es realmente la situación de la mujer. Se suponía que con una serie de proyectos y programas la mujer podía llegar a una igualdad con el hombre en el sentido más de incorporación a la fuerza de trabajo. Con la participación de algunas diputadas como Doña Carmen Naranjo como Ministra de Cultura, ... Tirza Rivera (ella era del Partido Unidad, que entonces tenía otro nombre, no existía la coalición), Alfonsina Chavarría, estaba Matilde Marín de Soto" (Entrevista realizada a Irma González, 17 de diciembre, 1996).

Con respecto al Proyecto de Ley de Igualdad de la Mujer tenemos como gestora a la Primera Dama de la República del período presidencial de 1986-1990, Margarita Penón:

"Quien convocó a dos destacadas mujeres, ex ministras de Estado y caracterizadas por su actividad en favor de los derechos de las mujeres: Carmen Naranjo y Elizabeth Odio. A quienes solicitó la elaboración de un Proyecto de Ley que diera sustento legal al objetivo de Igualdad Real propuesto por el Gobierno" (Moreno, 1995: 19). 
Después de ampliada la propuesta inicial, el Proyecto fue presentado en la Asamblea Legislativa el 8 de marzo de 1988 y fue aprobada dos años después, el 8 de marzo de 1990. En dicha ocasión fue apoyada por la mayoría de los diputados y diputadas, durante el proceso fue modificada, recortada y eliminados algunos de sus aspectos más polémicos, como eran las cuotas de participación política de las mujeres.

A su vez el Proyecto de Ley fue acompañado por un fuerte proceso de promoción que estuvo a cargo de la dirección del Centro Mujer y Familia, con el fin de contar con el apoyo de mujeres de distintos sectores sociales, dada la resistencia que tenían diputados(as) de ambos partidos mayoritarios del país para aprobar la Ley (Unidad Social Cristiana y Liberación Nacional).

Dicho proyecto, aunque en su momento contó con el apoyo de diversos sectores sociales de mujeres, no fue bien recibido por algunas agrupaciones feministas, quienes señalaban algunas debilidades y problematizaban los planteamientos del Proyecto de Ley. Para la Asesora Legal del Centro Nacional de Desarrollo de la Mujer y la Familia (Lic. Ana Elena Badilla) quien fue una de sus principales propulsoras y a quien le correspondió dar seguimiento a todo el proceso, considera que esta situación se explica por la coyuntura particular en que se encontraban algunas de estas agrupaciones y el tipo de preocupaciones que orientaban el trabajo, el cual, no necesariamente coincidía con la visión gubernamental:

"Yo creo que esta Ley no fue bien vista como una ley feminista, y quizá por ello algunas organizaciones femeninas caracterizadas como tales no dieron su apoyo a la misma. Fue una Ley concebida para trabajar por las mujeres desde una perspectiva de la igualdad, cuando en ese momento ya algunos sectores comenzaban a cuestionar el concepto de igualdad. ¿lgualdad con respecto a qué? ¿Cuál era el referente? Se rechazaba la idea de igualdad con respecto a los hombres" (entrevista a Ana Elena Badilla, realizada por Elsa Moreno en: Mujeres y política en Costa Rica, 1995: 24).

Vemos así, cómo aspectos nacionales se van relacionando y articulando con hechos internacionales que indudablemente tienen influencia en el proceso que se va gestando a nivel político y organizativo en nuestro país, como es la fundación y desarrollo del CMF y la gestión y aprobación del Proyecto de Igualdad Social de las Mujeres.

\subsection{Génesis y conformación del movimiento de mujeres en Costa Rica: rearticulando espacios de acción}

Los eventos de finales de la década del 70 fueron decisivos en el proceso de conformación del 
movimiento de mujeres costarricenses ya que generó un proceso de análisis de la situación de discriminación y exclusión estructural en la sociedad costarricense de las mujeres, así como por haber visto resurgir a las organizaciones feministas en nuestro contexto. Podemos al respecto señalar cuatro eventos significativos que se dieron en dicho período:

1. La celebración del Año Internacional de la Mujer, en 1975. El decreto del Decenio de las Naciones Unidas para la Mujer: Igualdad Desarrollo y Paz (1976-1985) y la Convención para la Eliminación de todas las formas de discriminación.

2. La constitución de grupos que se autodefinen como feministas. Este evento tiene gran transcendencia ya que en Costa Rica, después de la formación de la Liga Feminista en 1923, ninguna otra organización se había autoproclamado como tal. En este período se funda el grupo feminista llamado Movimiento de Liberación de la Mujer (MLM) (que surge al interior de la -OST Organización Socialista de los Trabajadoresen 1978) y el Grupo Ventana (1979). Ambos grupos se definen feministas y recibieron gran influencia de las corrientes feministas europeas. En términos generales, podemos afirmar que CEFEMINA nace como grupo feminista y desde sus inicios mostró gran identificación con los principios de la corriente liberal, sin embargo, su característica fundamental es la heterogeneidad ideológica interna. Por su parte Ventana se identificó más con la corriente feminista radical ${ }^{5}$.

3. La presencia e influencia que han tenido las diversas corrientes ideológicas feministas que se han gestado y desenvuelto en los países desarrollados de Europa, Canadá y Estados Unidos.

5 El movimiento de las feministas radicales surge en Estados Unidos durante la década del 60. Para ellas la opresión de las mujeres se origina primordialmente en sus relaciones íntimas, sexuales, y relativas a la procreación. Estas, además, de desarrollar la teoría del patriarcado, introdujeron aspectos relegados tanto a la vida privada como política. Plantean que toda relación entre hombres y mujeres supone relaciones de poder (Jaggar, 1983).

Las feministas culturalistas coinciden en varios aspectos con las radicales, en tanto parten también de que el sistema patriarcal oprime e impide el desarrollo de las mujeres. Pero las feministas culturalistas consideran que una tarea prioritaria a desarrollar es la recuperación y creación de una cultura femenina basada en principios femeninos. Tienden a separarse de la cultura dominante femenina en búsqueda de una cultura alternativa (Jaggar; 1983). 
4. La experiencia acumulada de las mujeres que participaron en diversas luchas de distintos sectores sociales. Muy rápidamente podemos señalar la participación de las mujeres que va desde la lucha en contra de la Dictadura de Los Tinoco (1917), pasando por la lucha Sufragista (1949, ya analizada con anterioridad), así como su participación en las Huelgas Bananeras y las luchas sindicalistas de principios del presente siglo, la Guerra Civil del 48 -participación totalmente invisibilizada- las luchas campesinas por la recuperación de tierras y las movilizaciones urbanas para la obtención de vivienda en la década del 60, así como la Huelga Nacional en contra del alza de las tarifas eléctricas en 1983 y las marchas campesinas del 87 y 88 (Morena, 1995). Todos estos hechos forman parte de los antecedentes del movimiento, en la medida en que la experiencia de las mujeres se va entrelazando, y las actoras de un movimiento, pueden ser a su vez, las gestoras del siguiente. Por supuesto, el proceso de desarrollo no es lineal, pero su influencia es vital.

A pesar de la transcendencia de los eventos de finales de los años 70, no fue sino hasta la década de los años 80s que el movimiento de mujeres costarricense logró constituirse como un movimiento social específico.

Hay varios factores que influyeron en el proceso de constitución del movimiento de mujeres en la década de los años 80 . Entre los factores más relevantes se pueden mencionar los siguientes:

1. Cambios agudos en las condiciones socioeconómicas de las mujeres costarricenses es el período de la crisis económica y del desarrollo de las políticas de ajuste estructural-.

2. La proliferación de muchas y diversas organizaciones de mujeres que se plantean como ejes de lucha el mejoramiento de las condiciones de vida de sus familias.

3. La presencia mayoritaria de mujeres en organizaciones reivindicativas mixtas y en importantes movilizaciones sindicales (ejemplo las luchas por vivienda).

4. La ampliación y diversificación de las organizaciones feministas, muchas de las cuales 
se conforman durante la década del 80 .

5. La constitución y fortalecimiento del movimiento de mujeres latinoamericano organizado y el desarrollo de las corrientes feministas en Latinoamérica, las que propiciaron el desarrollo de los Encuentros Feministas.

6. Con el desarrollo del movimiento de mujeres latinoamericano se propicia una afluencia de ideas político-ideológicas, así como la llegada de mucha literatura que influye en el quehacer político ideológico y organizativo de las experiencias nacionales.

\subsection{Grupos feministas y espacios de participación de las mujeres}

Las experiencias organizativas feministas en Costa Rica trascienden la coyuntura de la década del 80 estas son de gran valor para el desarrollo del movimiento feminista costarricense, como señalamos anteriormente. Sin embargo, el contexto de la década de los 80 influyó de forma decisiva en su desarrollo organizativo y en la ampliación de la base social, debido a dos razones.

1. En primer lugar, el establecimiento de relaciones entre las organizaciones feministas y diversas experiencias organizativas de mujeres de otros sectores sociales como: las mujeres organizadas en torno a las cooperativas, a las luchas por vivienda, al impulso de proyectos productivos en general y otras experiencias que van desde las eclesiales hasta las organizaciones campesinas.

2. En segundo lugar, en el contexto de la crisis económica se desarrollaron organizaciones feministas que se plantean como objetivo prioritario trabajar con mujeres de base (mujeres organizadas de los sectores populares) integrando tanto los problemas de clase, como los de género. Dicha perspectiva de trabajo dio origen a una nueva corriente dentro del feminismo costarricense: el feminismo popular. Los postulados de esta corriente fueron asumidos por organizaciones como la Alianza de Mujeres Costarricenses (a partir de 1987), La Carmen Lyra y el Colectivo de Mujeres Pancha Carrasco.

Por otra parte, a pesar de que no podamos establecer un vínculo directo entre la crisis económica y la presencia política de las mujeres para todos los períodos históricos, si debemos reconocer 
que estos dos elementos jugaron un papel decisivo en el desarrollo del movimiento de mujeres durante la década del 80. Sin embargo, debemos de tener presente que el factor de la crisis económica no fue la única variable importante en el desarrollo del movimiento de mujeres costarricenses.

La particularidad de los años 80 se define en función de la incorporación masiva de mujeres de distintos estratos sociales a diversas organizaciones, tanto como por la constitución de un número significativo de organizaciones de mujeres y organizaciones feministas. Debe tenerse en cuenta que en Costa Rica, al igual que en la mayoría de países de América Latina, las experiencias organizativas desarrolladas por las mujeres en ese período están relacionadas con actividades de subsistencia, autogestión de servicios y proyectos productivos.

A partir de los años 80 emergieron una gran cantidad de agrupaciones de mujeres ${ }^{6}$. Simultáneamente se fortalecieron algunos de los grupos feministas ya existentes: tal fue el caso del Centro Feminista de Información y Acción (CEFEMINA) y Alianza de Mujeres Costarricenses. También surgieron nuevos grupos feministas como el Colectivo de Mujeres Pancha Carrasco (1987) y la Asociación Carmen Lyra, y grupos lésbicos como La Colectiva Lésbica "Las Entendidas" (1986) y Las Humanas. Paralelamente se constituyen organizaciones que se plantean luchar por el derecho alternativo de las mujeres como el Comité Latinoamericano de Defensa de los Derechos de la Mujer CLADEM-Costa Rica (1988). CLADEM por su carácter supranacional potencia la coordinación regional.

En esta época también se dan las primeras experiencias de coordinación entre grupos de mujeres nacionales y regionales al calor de las actividades de solidaridad y en torno al proceso de Pacificación Centroamericana: la "Asamblea de Mujeres por la Paz Visitación Padilla", en 1989.

En los años 80 presenciamos la constitución de equipos de trabajo de mujeres líderes y activistas

6 Como anexo presentamos un listado de organizaciones de mujeres e instituciones que existen en nuestro país. Si se desea ampliar la información al respecto se puede consultar el Directorio que elaboró la Fundación Arias para la paz y el Progreso Humano: "Inventario de Organizaciones que trabajan con la Mujer en Centroamérica". Así mismo deseamos destacar la imposibilidad de contar con información específica sobre todos los grupos de mujeres que existen a nivel nacional ya que no existe un registro nacional. Solo contamos con información de cuantos grupos atienden algunas instituciones u organismos. Pero los datos concretos sobre el nombre, número de integrantes no están disponibles. 
al interior de las organizaciones gremiales mixtas como los sindicatos y las cooperativas ${ }^{7}$. Se organizan grupos comunales tanto urbanos como rurales alrededor de demandas prácticas de género. Proliferan los grupos productivos de mujeres que organizados por la vía cooperativista, asociativa o microempresarial, estimándose la existencia de unos 480 grupos de este tipo a mediados de los 80s (Quesada y Camacho, s.f.:5).

En 1988 se funda la Red de Mujeres Ecuménicas y Pastoras, la cual da origen a la Red de Mujeres Trabajando con Mujeres, donde participan mujeres de distintos sectores sociales. Se estima que esta Red llegó a integrar a unos 25 grupos de mujeres. En el Foro que realizó en 1990 se hicieron presentes 100 mujeres representantes de distintos sectores, entre ellos: mujeres campesinas, indígenas, pobladoras, académicas, feministas, y representantes de organismos diversos (ver memoria del Foro: Vida Cotidiana Impacto de la Crisis en la Mujer). Este evento permite observar el nivel de interrelación que se va construyendo entre los distintos grupos y sectores de mujeres.

La Red de Mujeres a pesar de su importancia tuvo una vida corta, malos entendidos entre los grupos y objetivos diversos imposibilitaron su desarrollo posterior. Un pequeño sector de dicha Red de Mujeres, liderada por el Colectivo Pancha Carrasco retoma la experiencia de coordinación, dándole esta vez un carácter más popular a la misma, dicha Red se dio a conocer como la Red de Mujeres en Acción.

Al día de hoy hay algunas organizaciones que ya no existen, tal es el caso de las Lésbicas y el Grupo Ventana. A pesar de su disolución, estas organizaciones cumplieron un papel relevante durante la década del 80 , ya que posibilitaron el desarrollo de actividades conjuntas y enriquecieron el debate en torno al papel que debían desempeñar las organizaciones feministas en el interior del movimiento de mujeres.

\footnotetext{
7 "A nivel sindical, en el marco de un Proyecto de la Organización Internacional del Trabajo, OIT de capacitación de mujeres sindicalistas, en 1985, se inicia la coordinación de mujeres sindicalistas de cuatro Federaciones: Central Unitaria de Trabajadores, CUT, Central de Trabajadores Costarricense, CTC, CCTD, CATD, cuyo objetivo fue propiciar la formación de comités de Acción Femenina al interior de los sindicatos, proceso que dio paso a la creación de las secretarías de la Mujer y al Comité Interconfederal Femenino -1986-1987-. En el sector cooperativo, en 1985 el IV Congreso del Movimiento Cooperativo se pronuncia con una resolución que establece como prioritario el trabajo de las mujeres. En 1987 surge Asociación Programa Nacional de Asesoría y Capacitación para la Mujer Cooperativista, APROMUJER" (Quesada y Camacho, s.f.:5).
} 
Por otra parte, resulta interesante observar las diferencias ideológicas en las distintas organizaciones. CEFEMINA (como ya se planteó antes) se caracterizó desde su origen por su heterogeneidad aunque con una importante influencia de principios del feminismo cultural. Las integrantes de "Ventana" se identificaban más con la corriente feminista radical. Mientras que el Colectivo Pancha Carrasco, la Carmen Lyra y Alianza de Mujeres se caracterizó por identificarse con la corriente del feminismo popular.

Estas diferencias a su vez se reflejaron en los distintos trabajos que desarrolló cada organización. De esta forma, Ventana se inclinó desde un inicio por grupos de autoayuda y socialización de su experiencia con mujeres de sectores medios (Facio, 1995).

CEFEMINA $^{8}$ acompañó el trabajo organizativo de COPAN y después empezó a desarrollar talleres sobre salud y grupos de autoyuda con las mujeres que participaron en los comités de lucha por vivienda y otras que se acercan a la organización.

Por otro lado, Alianza de Mujeres Costarricense y la Carmen Lyra se dedicaron a seguir desarrollando el trabajo que realizaron décadas atrás desde el Partido Vanguardia Popular, pero empezaron a enfatizar en las situaciones de discriminación que se sufre no solo por las condiciones de clase, sino también por las de género. Alianza de Mujeres también se ha caracterizado por el desarrollo de un trabajo sistemático en torno a los derechos legales de las mujeres y la defensa de éstos. Estas dos organizaciones a pesar de que se ubican dentro de la misma corriente que el Colectivo de Mujeres Pancha Carrasco se diferencian de éste porque el Colectivo Pancha Carrasco siempre puso más énfasis en las condiciones de género de las mujeres que en las de clase ${ }^{9}$.

Por otra parte, en la segunda mitad de la década del 80 las feministas del Partido Liberación Nacional empezaron a plantear soluciones a problemas específicos de las mujeres. En 1988 desarrollaron claras acciones en pro de la defensa de los derechos de las mujeres. Apoyaron con gran fuerza el programa de las 80000 viviendas y se organizó, desde la oficina de la Primera

8 Los datos que en adelante se señalan sobre el trabajo que realizaron las organizaciones feministas se obtuvo del Inventario de Organizaciones que trabaja con la Mujer en Centroamérica. Editado por la Fundación Oscar Arias para la Paz y el Desarrollo. 1993.

9 El Colectivo Pancha Carrasco a partir de 1993 se autoproclama como feminista y no como feministas populares (Quesada y Camacho, 1993). 
Dama, la propuesta del Proyecto de Igualdad Real de la Mujer, con el respaldo del Centro Nacional para el Desarrollo de la Mujer y la Familia.

Por último, debemos tener presente que los grupos feministas se acercan a apoyar experiencias organizativas de mujeres de sectores populares después de fundadas o creadas éstas, ya que se evalúa como positiva la fuerte presencia de las mujeres en estas organizaciones y se visualiza la posibilidad de extender y compartir con otras mujeres reivindicaciones que habían sido solo acuerpadas por las feministas en períodos anteriores. ¡Error! No se encuentra el origen de la referencia.

\subsection{Desarrollo y coordinación del movimiento de mujeres en los noventas}

Durante la década de los años 90 , los organismos no gubernamentales (ONGs) ${ }^{10}$ y las organizaciones de base de las mujeres amplían sus espacios de participación. Las ONGs se diferencian de las organizaciones de base en la medida en que las primeras en su mayoría están formadas por mujeres profesionales, reciben apoyo económico de la cooperación internacional, razón por la cual en los últimos años se han institucionalizado. Los grupos de base por el contrario son organizaciones formadas por mujeres de sectores populares, se organizan para mejorar sus condiciones de vida, no reciben salario, sino que su trabajo es voluntario a no ser que independiente de su trabajo organizativo cuenten con fondos para ejecutar algún proyecto (en su mayoría) productivo.

En este período, surgen muchos grupos de base, urbanos, rurales e indígenas. Las mujeres de muchos sectores sociales se incorporan a participar en grupos de mujeres y a través de programas que desarrollan las ONGs y el gobierno (principalmente a través del Centro de Desarrollo Nacional de la Mujer y la Familia, aunque pueden contar a su vez con el apoyo de alguna o varias organizaciones feministas, (ONGs $u$ otras instancias de bienestar social) tienen acceso a talleres de capacitación en aspectos relativos al género, condición y posición de las mujeres en la sociedad, etc.

\footnotetext{
10 Estos organismos son comúnmente conocidos bajo el concepto de ONGs, las cuales se caracterizan por ser instituciones autónomas que reciben fondos de la cooperación internacional con el fin de desarrollar proyectos de bienestar social. Durante la última década una de los sectores prioritarios de la cooperación internacional han sido las mujeres, razón por la cual se ha logrado crear y consolidar espacios de trabajo de las mujeres, que desarrollan trabajo con mujeres de distintos sectores sociales, principalmente con mujeres de escasos recursos económicos.
} 
A su vez, las organizaciones de base, las ONGs nacionales, así como organismos internacionales participan en espacios que les permiten desarrollar acciones de forma coordinada, tanto en el nivel nacional como regional o bien internacional. Las acciones que se desarrollan entre distintos grupos que forman parte del movimiento de mujeres tiene a su vez como interlocutor el Estado. Es un período en el cual el movimiento de mujeres ha tenido posibilidad de coordinar propuestas o bien negociar el desarrollo de algunas acciones. Ello ha sido importante y significativo ya que el proceso de desarrollo del movimiento de mujeres llega a tal punto que el gobierno de turno debe tener como punto de referencia al movimiento de mujeres -y en especial al movimiento feministapor ser un sector social importante, en la medida en que el mismo ha mostrado capacidad organizativa.

La década de los años 90 se caracteriza además por la diversificación de los espacios de coordinación, el cual se da tanto a nivel nacional como regional (centroamericano y latinoamericano). Las relaciones y las alianzas se complejizan tanto al interior del movimiento de mujeres, como hacia el exterior, es decir entre el movimiento y el Estado:

"Se notan también cambios significativos de incorporación de feministas a espacios institucionales y de coordinación, donde logran aportar, dinamizar y concretar algunos de sus planteamientos y propuestas" (Camacho y Flores, 1987: 464).

Entre los espacios de coordinación de mayor importancia se puede mencionar:

$\hat{\imath}$ La Colectiva 25 de noviembre: Es un espacio de coordinación cuyo principal fin era la organización de la marcha nacional que se organiza el 25 de noviembre, fecha en que se celebra el día Internacional contra la violencia hacia las Mujeres. Surge en 1991.

$\hat{\imath}$ La Colectiva Feminista Nacional: Fue uno de los primeros espacios de carácter colectivo que posibilitó la participación y coordinación entre distintos grupos de mujeres. Desde dicho espacio las mujeres se abocaron a la organización del Encuentro Nacional de Mujeres: "Mujer y Poder" realizado en Santa Ana, en noviembre de 1992. A su vez dicho Encuentro sirvió como actividad previa al I Encuentro Centroamericano de Mujeres: "Una Nueva Mujer un Nuevo Poder" (realizado en marzo de 1993). Dicho Encuentro Regional a su vez formó parte de las acciones preparativas del "VI Encuentro Feminista Latinoamericano y del Caribe", el cual fue organizado por el movimiento de mujeres Centroamericano y realizado en El Salvador 
en noviembre de 1993. Este Encuentro forma parte de los periódicos Encuentros Feministas Latinoamericanos y del Caribe que se realizan desde finales de los años 70.

A su vez se realizan iniciativas puntuales las cuales tienen un objetivo más coyuntural como:

$\hat{y}$ La instancia de coordinación que giró en torno a la defensa de los derechos de las humanas cuyo interés fue desarrollar acciones preparatorias a la Conferencia Mundial sobre Derechos Humanos realizada en 1992, en Viena. En dicho proceso participaron organismos como: El Comité Latinoamericano de Defensa de los Derechos de la Mujer, CLADEM-Costa Rica, Instituto Latinoamericano de Naciones Unidas, ILANUD, Comisión de Derechos Humanos de Centro América, CODEHUCA, Instituto Iberoamericano de Derechos Humanos, IIDH y Radio Feminista Internacional, FIRE.

今 En 1994 se creo otro espacio de coordinación en el cual participan organismos no gubernamentales, gubernamentales y organizaciones de mujeres que están interesadas en elaborar y promover un Proyecto de Ley para prevenir y sancionar la violencia contra las mujeres.

$\hat{y}$ Se creó otro comité cuya temática de interés ha girado en torno a los derechos sexuales y reproductivos, dicho espacio se fundó para desarrollar acciones preparatorias a la Conferencia de Población y Desarrollo que se realizó en El Cairo en 1994. Luego de la actividad se realizaron actividades de seguimiento, sin embargo, este proceso en alguna medida fue sustituido por las iniciativas que giraron en torno a la Cuarta Conferencia Mundial de la Mujer llevada a cabo en agosto de 1995. Para dicha actividad se creo el espacio de coordinación llamado "La iniciativa nacional de Beijing". Su función fue la de coordinar regionalmente el proceso organizativo previo a la Conferencia Mundial, llevar propuestas y análisis críticos de los temas que se desarrollarían en la Conferencia Oficial de las Naciones Unidas. Después de efectuada la Conferencia la instancia de coordinación cambió el nombre por "Iniciativa de las Mujeres para el Cumplimiento de la Plataforma de Acción" (PAN) (Camacho y Flores, 1997).

$\hat{y}$ La creación del Comité Intersectorial, que se fundó con el objetivo de facilitar la coordinación regional, con el fin de lograr mayores niveles de incidencia en el proceso previo y posterior a 
la Conferencia Oficial de las Mujeres de las Naciones Unidas. Actualmente se conoce más como el Comité Post-Beijing.

Otra característica importante de la década de los años 90 ha sido el surgimiento de nuevas organizaciones de mujeres como el Consejo de Mujeres Indígenas, la Asociación de Mujeres Afrocaribeñas, la Asociación Nacional de Mujeres con Discapacidad, iniciativas de mujeres jóvenes por desarrollar actividades específicas de ese sector como el Grupo de Mujeres Antítesis, la Red de Mujeres en Acción, la Asociación Nacional de Madres Comunitarias, la Fundación FEMINARIA, entre otras.

A su vez contamos con la creación de redes temáticas a nivel centroamericano, tal es el caso del Programa la Corriente creado en 1993, el espacio Iniciativa a Beijing creado en 1994 y la Red Centroamericana contra la violencia en 1995.

En palabras de las autoras Camacho y Flores quienes realizaron una investigación sobre el movimiento de mujeres en Costa Rica y con las cuales coincidimos, el escenario del movimiento de mujeres ha tenido como puntos focales de interés las cinco Conferencias Mundiales de las Naciones Unidas realizadas durante la década del noventa: Medio Ambiente y Desarrollo (Río de Janeiro, 1992), Derechos Humanos (Viena, 1993), Población y Desarrollo (Cairo, 1994), Desarrollo Social (Copenhague, 1995) y la Cuarta Conferencia Mundial de la Mujer (Beijing, 1995).

Después de realizada la Cuarta Conferencia Mundial de la Mujer los grupos de mujeres que se aglutinaron en torno al Comité Intersectorial y a la iniciativa Nacional hacia Beijing, realizaron actividades de evaluación. En dichas actividades se analizó el papel desempeñado en la Conferencia por la representación nacional y los conflictos que se derivaron de las diferencias entre el movimiento de mujeres y la representación oficial. Dichas diferencias generaron un distanciamiento entre el movimiento de mujeres y el CMF entidad que formaba parte de la representación oficial. A pesar de las diferencias políticas entre ambas partes debemos destacar los esfuerzos que realizaron para darle continuidad y establecer mecanismos de control para la ejecución de acciones y desarrollo de políticas que quedaron establecidas en el documento final de la Conferencia. 
Por último deseamos destacar el desarrollo de un espacio de coordinación de reciente creación llamado La Agenda Política de las Mujeres (1997). Iniciativa que surge con el fin de contar desde el movimiento feminista con una agenda propia que le posibilite negociar y ser interlocutora de los partidos políticos previa y posteriormente a las elecciones nacionales así como interpelar al Estado en asuntos relativos a las mujeres, con el fin de negociar aspectos estratégicos de las mujeres que puedan ser incorporados en los programas de gobierno. Este espacio de coordinación lo integran principalmente mujeres profesionales que participan en él a título personal. Actualmente lo integran aproximadamente 40 mujeres que están a su vez desarrollando proyectos en pro de la defensa de los derechos de las mujeres desde las instituciones $u$ organismos en que laboran. (Entrevista: Yamileth Ugalde quien fungía como promotora de Agenda Política de las Mujeres, en junio 1998).

\section{ALGUNOS DE LOS NUDOS DEL MOVIMIENTO DE MUJERES COSTARRICENSES}

El proceso de desarrollo del movimiento de mujeres que hemos esbozado en las páginas anteriores nos permite identificar algunas de las acciones realizadas para defender sus derechos.

Vemos como en un primer período, que va desde finales de los años 70 y cubre la década de los años 80 , el movimiento de mujeres realiza acciones más en función de su desarrollo como tal. Ese "ensimismamiento" tiene que ver con la necesidad de reafirmación de su independencia, autonomía y derecho a ejercer nuevas formas de hacer política.

En un segundo período adentrándose en la década del 90, el movimiento debe enfrentar nuevas coyunturas, nuevos problemas, nuevas necesidades, producto de su desarrollo y gestión. La creciente presencia del movimiento de mujeres, en especial las organizaciones feministas en el ámbito público, lo llevó a repensar sus relaciones con el Estado (Guzmán, 1994). Consecuentemente el Estado se convierte en un interlocutor del movimiento de mujeres; en la medida en que éste demanda derechos, negocia por el desarrollo de programas que integre una perspectiva de género y lucha por la distribución equitativa de sus recursos.

En este nuevo período el Estado es visto por el movimiento de mujeres como un espacio de poder - por lo que es importante acceder a él-, pero por su carácter tiende a canalizar el dinamismo de los actores y actoras sociales, debilitando muchas veces su capacidad subversiva, cooptando el 
movimiento, e institucionalizando sus demandas. Riesgo que ha empezado a ser planteado recientemente por algunos sectores del movimiento de mujeres, y que por la importancia del mismo se ha convertido en uno de sus nudos ${ }^{11}$ centrales.

Otros nudos a los que se enfrenta el movimiento de mujeres costarricense tiene que ver con la construcción de autonomía, la política de alianzas, la diversidad del movimiento, la representación y los recursos financieros. A continuación nos referiremos a los nudos de la autonomía y la política de alianzas.

Durante los años 80s las feministas y mujeres del movimiento en general enfatizaron sobre la necesidad de construir de forma autónoma nuevos espacios organizativos de mujeres. Dicha necesidad se fue construyendo paulatinamente en los diferentes espacios, como cooperativas, partidos políticos, sindicatos, organizaciones campesinas, en organismos institucionales y organismos no gubernamentales, así como la creación de organizaciones de mujeres. Dicho planteamiento fue muy debatido y poco apoyado por participantes de las distintas expresiones organizativas -sobre todo varones, aunque también algunas mujeres asumieron esa misma posición- temían la división interna producto de la creación de espacios específicos de mujeres.

El nudo de la autonomía ha variado, en la medida en que el movimiento de mujeres se ha desarrollado. Hoy día, además de la defensa y construcción de espacios autónomos al interior de distintas organizaciones, la autonomía se plantea también con respecto al Estado, a los programas gubernamentales e incluso se habla de autonomía de grupos de mujeres con respecto a otros grupos de mujeres -sobre todo con respecto a las ONGs o bien organismos que trabajan y apoyan los grupos de base-.

Por lo tanto, podríamos decir que el movimiento de mujeres ha pasado de una autonomía "defensiva", a una autonomía propositiva. El cambio se explica por el nuevo contexto en el que se actúa. Se trata ahora del contexto de la globalización, del desarrollo del modelo neoliberal, es

\footnotetext{
11 Dicho concepto ha sido ampliamente usado por el movimiento de mujeres, hace referencia a las dificultades que enfrenta el movimiento de mujeres y el feminista. Los nudos con el paso del tiempo se han ido complejizando y ampliando, y responden al interés de las mujeres por reflexionar sobre su practica política. Julieta Kirkwoord, plantea que los nudos del movimiento de mujeres aluden a la forma de crecimiento del mismo, *la cual es ni suave, ni armónico. Dice que a ellos nos podemos acercar apresuradamente, tratando de eliminarlos con un tajo de espada, como hizo Carlo Magno con el nudo Gordiano, para de esa forma eliminar la búsqueda o la discusión. Por otro lado, se puede intentar desenredarlos, separar sus hilos, buscando sus inicios, seguir sus entrelazamientos y sus 
también el contexto en el cual algunas de las demandas de las mujeres ha sido asumidas por los gobiernos y la sociedad civil, es el contexto de las Conferencias Mundiales -que exigen respuestas y elaboración de propuestas de parte de las mujeres "por ello aunque no se ha cambiado todo lo que debería haberse cambiado, las condiciones para el desarrollo de nuestras políticas han cambiado" (Vargas y Olea, 1998). Actuar como sujeta social que ya no solo demanda y exige, sino que debe a su vez proponer, le plantea nuevos retos al movimiento.

Este nuevo momento ha permitido visualizar debilidades del movimiento de mujeres de nuestro país, en el cual, desde nuestra apreciación, pesa la individualidad, opacando el quehacer colectivo; los liderazgos individuales, sobre los liderazgos compartidos; los intereses personales sobre los del grupo. Dicha problemática nos lleva al nudo de las alianzas y la diversidad del movimiento.

El nudo de las alianzas ha sido importante en el proceso de desarrollo del movimiento de mujeres. Conforme se pasa de la política de la confrontación a la política de elaboración de propuestas y a la negociación, es necesario generar alianzas entre las diferentes expresiones del movimiento de mujeres, con el fin de ganar terreno y potenciar las mismas. Tratar de impulsar propuestas colectivamente ha llevado al movimiento de mujeres a analizar cuáles son temas prioritarios y estratégicos, lo cual es complejo en un medio que se caracteriza por su heterogeneidad. Debido a que los puntos de vista son diversos, las necesidades e intereses no siempre son los mismos, estamos así frente a la encrucijada de la diversidad versus la universalidad.

En la medida en que se acepta que no todas las mujeres son iguales, dado las diferencias étnicas, de edad, posición política, económicas, raciales, nos enfrentamos ante la existencia de múltiples identidades. Ello ha llevado a que se dé la fragmentación del movimiento, de ahí la necesidad actual de desarrollar políticas de alianzas que acerquen a distintos y heterogéneos sectores, para conjuntamente establecer agendas de trabajo, a partir de la negociación y el diálogo.

Siguiendo a Vargas y Olea podemos decir que uno de los retos del movimiento está en desarrollar agendas de trabajo que den respuesta a las múltiples indentidades con el fin de evitar políticas de identidad excluyentes, con verdades incomunicables o transmitibles solo a los y las

reacomodos, para Julieta Kirkword, a través de los nudos diminutos, se va conformando la política feminista (Vargas y Olea,1998). 
iniciadas. Programas rígidos, según Hobswand "no son programas para hacer frente a los problemas del siglo XX sino es más bien una reacción (y lastre) emocional” (Vargas y Olea, 1998: 8).

Por otra parte, señalan Camacho y Flores que la mayor debilidad que tiene el movimiento de mujeres de nuestro país para desarrollar políticas de alianza, tanto al interior del movimiento como con agentes externos -Estado, instituciones, Conferencias Mundiales-, es su alto nivel de despolitización. Sostienen estas autoras que:

"este fenómeno, tan peculiar a nuestra identidad, incide directamente en la construcción de estrategias políticas. De manera que las alianzas se inscriben en un plano táctico y coyuntural, en lugar de una dimensión estratégica política del Movimiento... Incide a su vez el bajo nivel de desarrollo político -- organizativo, aunada dicha debilidad al déficit histórico de las mujeres en cuanto a la política del pacto" (Camacho y Flores, 1997: 479).

Desde nuestro punto de vista, no es la despolitización o el bajo desarrollo político organizativo, lo que explica la debilidad que tiene el movimiento de mujeres para desarrollar políticas de alianza. A nuestro parecer el principal impedimento es la existencia de una cultura de la intolerancia. Pasar del discurso del "respeto de las diferencias" a la práctica es uno de los retos del movimiento de mujeres de nuestro país. Sin embargo, consideramos que desarrollar dicha actitud es parte del proceso de trabajo del movimiento de mujeres, del aprendizaje, en la medida en que la misma coyuntura llama al acercamiento y al ejercicio cotidiano de estas alianzas, pactos o procesos de coordinación. La Realización de las Conferencias de las Naciones Unidas, y los procesos de trabajo del movimiento de mujeres a nivel nacional, regional y mundial contribuyen al acercamiento de las organizaciones de mujeres, llamándolas en la práctica a negociar, pactar y desarrollar alianzas al menos coyunturales.

\section{CONCLUSIONES}

A pesar de la complejidad del concepto de cultura política y de sus múltiples conceptualizaciones, esta conceptualización presenta un sesgo al no incluirse la diferenciación entre la cultura política de los hombres y la de las mujeres. Los análisis al tener una perspectiva androcéntrica no reflejan 
adecuadamente la participación de las mujeres en sus estudios.

El trabajo que hemos realizado nos permite observar para el caso de Costa Rica cómo las mujeres han tenido una fuerte e importante participación política en distintos espacios. Ello contradice la visión tradicional que existe hacia la participación político organizativa de las mujeres desde el análisis de la cultura política. El trabajo nos permite visualizar como las mujeres no solo participan en procesos políticos de gran envergadura, sino que trae consigo procesos de cambio, que por lo demás no podríamos tan siquiera relacionarlos con actos conservadores. Todo lo contrario, demuestran su capacidad organizativa y el impulso al cambio que generan con sus propuestas político, sociales, culturales y organizativas.

Podemos decir que los primeros antecedentes del feminismo en Costa Rica datan de las últimas décadas del siglo XIX. Se trató de experiencias que estuvieron integradas y dirigidas por grupos de mujeres profesionales y del sector medio y que se identificaron con el pensamiento liberal de la época.

Las primeras organizaciones de mujeres se desarrollaron en Costa Rica desde fines del siglo XIX, con el surgimiento de las luchas sufragistas de las mujeres de la época. Durante la primera mitad del siglo XX se fundaron otras organizaciones de mujeres como la Liga Feminista y la Alianza de Mujeres Costarricense.

Sin embargo; estas experiencias no llegaron a constituir un movimiento de mujeres en tanto se mantuvieron como expresiones organizativas que restringieron su participación a sectores de la población muy definidos. En las sufragistas participan mujeres de sectores medios-altos y en la Alianza de Mujeres los sectores populares. Así mismo, estas organizaciones no coinciden en el tiempo, corresponden a períodos históricos distintos. Por su parte, las mujeres del Partido Liberación Nacional durante las décadas de los años 50 a los años 70 se dedican principalmente a aspectos políticos del partido.

Por esas razones y por la forma en que se desarrollan los hechos en la década de los años 80 es 
que podemos afirmar que el movimiento de mujeres en Costa Rica se constituye a partir de esa década. Es en los 80 cuando surge un movimiento multisectorial que se desarrolló con gran dinamismo y vitalidad y que se diferencia radicalmente de las experiencias previas que son más particulares, logrando, por primera vez, un nivel de articulación y relaciones entre distintas experiencias organizativas. Cuenta, además, con un referente de identidad propio, surgido como resultado del análisis de las necesidades e intereses de las mujeres, el intercambio de opiniones entre los grupos nacionales y entre estos y grupos internacionales, y por supuesto, al calor de las luchas y demandas realizadas.

Durante la década de los años 80, el movimiento feminista costarricense experimentó un significativo desarrollo. Este desarrollo fue producto del fortalecimiento de los grupos existentes (como Alianza de Mujeres, CEFEMINA), y del nacimiento de nuevas organizaciones que se autodefinen como feministas (Colectivo de Mujeres Pancha Carrasco; Carmen Lyra, entre otros). También contribuyó al fortalecimiento del movimiento feminista costarricense, los esfuerzos que realizaron estas organizaciones por relacionarse con los grupos de mujeres de base constituidos en ese mismo período. El entramado de relaciones que se establece entre las múltiples agrupaciones de mujeres permite relevar temas de trascendencia nacional, cuya lucha requiere la participación activa de múltiples organizaciones y personas. Es decir, se generan en dicho período demandas y luchas que desbordan la capacidad operativa de cada uno de los grupos y que exigen el establecimiento de vínculos y alianzas entre las diferentes agrupaciones, lo cual es indicativo de la emergencia del movimiento social de mujeres en tanto tal.

La década de los años 80 también se caracteriza por la constitución de un gran número de grupos de mujeres pobladoras y de otros sectores sociales. Estas organizaciones tenían como objetivo principal mejorar la condición de vida de las familias. El vínculo que se establece entre muchas de estas agrupaciones y las organizaciones feministas o que laboran con enfoque de género permiten, de manera paulatina, a varias organizaciones de pobladoras, campesinas, cooperativistas $\mathrm{u}$ otras, integrarse en el movimiento de mujeres, compartir sus luchas y reivindicaciones. En ese sentido se generan nuevos referentes de identidad, permeados en este caso, por los referentes de identidad propios del movimiento de mujeres.

En síntesis se puede afirmar que los años 80s marcan un punto de ruptura positivo en la constitución del movimiento de mujeres costarricense. Por primera vez mujeres de sectores populares se 
organizan de múltiples formas en diversas organizaciones y en gran cantidad, para plantear sus necesidades, denunciar el carácter patriarcal de la sociedad costarricense y exigir el desarrollo de transformaciones sociales, culturales, políticas y económicas que le permitan a las mujeres vivir dignamente. Además en dicho período se produjo el encuentro orgánico entre las organizaciones típicamente feministas y las organizaciones de mujeres populares, en particular las pobladoras. Encuentro que a su vez ha generado conflictos y contradicciones al interior de ambas partes; contradicciones que aún no han sido superadas ni enfrentadas adecuadamente por parte del movimiento de mujeres. Sin embargo, dicho encuentro ha permitido combinar algunas de las reivindicaciones prácticas de género con estratégicas de género, condición indispensable para la conformación de los grupos de mujeres en un movimiento social. 


\section{BIBLIOGRAFÍA}

Ardaya, Gloria. "Mujeres y democracia. En busca de una identidad en el sistema político". En: Nueva Sociedad; Venezuela, nov.-dic. 1994, №134.

Astelarra, Judith y Amorós, Celia. "Cultura política de las mujeres". En: $\underline{\text { Cuadernos de Ciencias }}$ Sociales no.40, FLACSO. Costa Rica; 1991.

Cisneros, César; Sánchez, José y Badie, Bertrand. "Cultura política". En: Cuadernos de Ciencias Sociales. no.75, FLACSO, Costa Rica; 1994.

De Oliveira, Orlandina. "Presencias y ausencias femeninas". En: Trabajo Poder y sexualidad. México, El Colegio de México, 1991.

Diez, Rosa. "El desafío de la participación social". En: Isis Internacional no.13; Chile. Ediciones de la Mujer, 1990.

Escalante, Cecilia; Barahona, Macarena y Guzmán, Laura. "Balance sobre la situación de la mujer en la política en Costa Rica". (s.f.).

FLACSO. Mujeres Latinoamericanas en cifras: Costa Rica. Madrid. Instituto de la Mujer de Madrid y FLACSO Chile, 1993.

García, Ana Isabel y Gomáriz, Enrique. Mujeres Centroamericanas: Efectos del Conflicto. San José, Costa Rica. FLACSO, vol. 2, 1989.

IIDES. "Las españolas ante la política". España, Ministerio de Asuntos Sociales, Instituto de la Mujer. Serie Estudios № 21, 1988.

Lara, Silvia. "Feminismo y cambio social: El caso de las mujeres dirigentas en la lucha por vivienda en Costa Rica". Tesis para optar al grado de Magister Scientiae. Universidad de Costa Rica, Maestría Centroamericana de Sociología, 1994.

Lycklama á Niejeholt, Geertje, Vargas, Virginia y Wieringa, Saskia. Womens movements and public policy in Europe, Latin America, and the Caribbean. Estados Unidos: Garlans Publishing, 1998.

Moran, Mํㅡㄹ Luz. "La cultura política de las españolas". En: Isis Internacional; Ediciones de la Mujer № 13, 1990.

Pérez, Dellanira y Profitt, Norma. Tiré el silencio afuera: sistematización de la experiencia de un grupo de apoyo mutuo y reflexiones sobre la violencia contra la mujer. San José, C.R.. Colectivo de Mujeres Pancha Carrasco, 1992.

Rodríguez, Regina; Portugal, Ana Ma y Saa Ma Antonieta. "Entre la democracia y la utopía". En: Isis Internacional. Chile, Ediciones de las Mujeres № 13, 1990.

Quesada, Liliana y Camacho, Lorena. Del feminismo popular al feminismo como opción vital y 
política. San José, Costa Rica. Colectivo de Mujeres Pancha Carrasco. s.f.

Red de Mujeres Trabajando con Mujeres. Memoria Foro: Vi da cotidiana e impacto de la crisis en la mujer. San José, Costa Rica, 1990.

Sagot, Monserratt. "Aquí yo he aprendido a luchar". En: Casa de la Mujer.Instituto de Estudios de la Mujer, Universidad Nacional Autónoma. No.6, dic. 1994.

Vargas, Virginia. Cómo cambiar el mundo sin perdernos: El movimiento de mujeres en Perú y en América Latina. Perú: Ediciones Flora Tristán, 1992.

“Ciudadanía”. Lima, 1997 (Mimeo).

Vargas , Virginia y Olea, Cecilia. "Los nudos de la Región", 1996. (Mimeo síntesis de la IV Conferencia Mundial de la Mujer), Beijing.

Nancy Piedra Guillén

Escuela de Sociología

Facultad de Ciencias Sociales

Universidad de Costa Rica 